\title{
Pinning Two Nonlinearly Coupled Complex Networks with an Asymmetrical Coupling Matrix
}

\author{
Jianwen Feng, Ze Tang, Jingyi Wang, and Yi Zhao \\ College of Mathematics and Computational Sciences, Shenzhen University, Shenzhen 518060, China \\ Correspondence should be addressed to Jianwen Feng; fengjw@szu.edu.cn
}

Received 15 September 2012; Accepted 18 November 2012

Academic Editor: M. De la Sen

Copyright (c) 2013 Jianwen Feng et al. This is an open access article distributed under the Creative Commons Attribution License, which permits unrestricted use, distribution, and reproduction in any medium, provided the original work is properly cited.

\begin{abstract}
This paper addresses the hybrid synchronization problem in two nonlinearly coupled complex networks with asymmetrical coupling matrices under pinning control schemes. The hybrid synchronization of two complex networks is the outer antisynchronization between the driving network and the response network while the inner complete synchronization in the driving network and the response network. We will show that only a small number of pinning feedback controllers acting on some nodes are effective for synchronization control of the mentioned dynamical networks. Based on Lyapunov Stability Theory, some simple criteria for hybrid synchronization are derived for such dynamical networks by pinning control strategy. Numerical examples are provided to illustrate the effectiveness of our theoretical results.
\end{abstract}

\section{Introduction}

Complex networks become more and more important because they abound both in nature and in the artificial networks (easy examples include biological ecosystems, internet connections, the World Wide Web, and various social and neural networks $[1,2])$. Complex networks also provide models for many large-scale and complicated systems occurring in nature and society that would otherwise be extremely difficult to study. Indeed, complex networks have been extensively studied over the past two decades in many fields of science and engineering because of their general ability to represent virtually all natural and man-made structures. The focus of most of these previous works, however, have been on the structural properties of the dynamical networks [14] with little regard for their collective behavior which is where some interesting phenomena such as synchronization, spatiotemporal chaos, autowaves, and spiral waves $[5,6]$ occur. Synchronization, in particular, is the time-correlated behavior between the nodes of a dynamical network and it is a collective behavior of coupled nonlinear systems that promises immense potential engineering applications [7-12].
Many synchronization patterns have been recorded in the literature such as complete synchronization [7-10], $\mu$ synchronization [11], cluster synchronization $[13,14]$, generalized synchronization [15], lag synchronization [16, 17], and phase synchronization [18] and it is known that they can all be encompassed by a unified definition [19]. One particular type of generalized synchronization that occurs commonly in symmetrical oscillators is antisynchronization (AS). Specifically, AS is characterized by the vanishing of the sum of the relevant variables and is a phenomenon that has both been experimentally and numerically observed in coupled chaotic systems. AS has been observed, for example, in piecewise linearly coupled Chua's circuits [20] and in coupled laser systems [21] and it is an important phenomenon in chaotic systems that has great application potential. Using antisynchronization in lasers, for instance, one can generate not only drop-outs of the intensity with ordinary low frequency fluctuations but also short pulses of high intensity that provides a new way of generating pulses of special shapes. Communications security and secrecy can similarly be enhanced during transmission by continuously transforming the digital signals between the synchronized 
and antisynchronized modes. Indeed, antisynchronization has been fairly well studied and many results exist about the phenomenon. An adaptive control method, for example, was proposed in [22] for antisynchronizing an uncertain Chua's chaotic system and sufficient conditions for both the synchronization and antisynchronization of the Colpitts oscillators were obtained using active control in [23]. In another attempt, a nonlinear control technique was used to antisynchronize two distinct chaotic systems in [24] and several sufficient conditions were obtained for the attainment of AS and adaptive AS in linearly coupled systems in [25]. The antisynchronization of a class of delayed chaotic neural networks was also investigated in [26, 27] using the Halanany Inequality and Lyapunov Stability Theory.

Recently, there is much interest in the coexistence of antisynchronization and complete synchronization (called hybrid synchronization) in chaotic systems [28-31] although much of these studies have been concerned with coupled chaotic systems rather than with coupled networks. The only investigation of the hybrid synchronization of two-coupled complex networks, to date, was that carried out by Sun et al. in [32] in which linearly coupled complex networks (i.e., the coupling function being linear) were considered. The fact remains, however, that many state variables of a network could at times be totally unobservable with $h\left(x_{i}(t)\right)$ (which is a nonlinear function of the state [32]) being the only known quantity.

Since it is literally impossible to add controllers to all nodes, local feedback injections are applied to only some of the nodes (this is known as pinning control). It has been revealed that, in the process of controlling various networks, pinning control serves as a simple and effective strategy for stabilization and synchronization. Pinning state feedback controllers have recently been used for the synchronization of complex dynamical networks by controlling a small fraction of nodes [33-35]. The pinning strategy has been shown to be effective for synchronization of networks. In this paper, we investigate the hybrid synchronization of nonlinearly coupled complex networks that are described by ordinary differential equations (ODE) with coupling functions that are nonlinear and whose coupling matrix is asymmetrical. Some simple criteria are derived for the hybrid synchronization of such dynamical networks by using the pinning control method and the Lyapunov stability theory. The results of this paper are a nontrivial extension of those in [32], where the coupling function is linear and the controllers were added to all nodes for getting hybrid synchronization.

This paper is organized as follows. In Section 2, we construct the hybrid synchronization of nonlinearly coupled complex networks with differential drive and response network local dynamics. In Section 3, we give some necessary definitions, lemmas, and hypotheses. In Section 4, we derive some simple criteria for the hybrid synchronization of such dynamical networks by pinning control using Lyapunov stability theory. In Section 5, we present some numerical simulations that validate the theoretical results. This paper concludes in Section 6.

\section{Model Description}

In [32], the authors used linear feedback or adaptive linear feedback methods to realize the hybrid synchronization of two-coupled networks. The driving network is

$$
\dot{x}^{i}(t)=f\left(x^{i}(t), t\right)+c \sum_{j=1}^{N} a_{i j} \Gamma x^{j}(t), \quad i=1,2, \ldots, N,
$$

and the response network is

$$
\begin{array}{r}
\dot{y}^{i}(t)=f\left(y^{i}(t), t\right)+c \sum_{j=1}^{N} b_{i j} \Gamma y^{j}(t)-d_{i} \Gamma\left(x^{i}+y^{i}\right), \\
i=1,2, \ldots, N .
\end{array}
$$

For the coupling system (1), we let the inner coupled matrix $\Gamma$ to be the identity matrix. Then the driving network of the nonlinearly coupled differential equation is the following:

$$
\dot{x}^{i}(t) f\left(x^{i}(t), t\right)+c \sum_{j=1}^{N} a_{i j} h\left(x^{i}(t)\right), \quad i=1,2, \ldots, N .
$$

In the following, the hybrid synchronization of the nonlinearly coupled complex network model (3) is investigated. To realize the hybrid synchronization, some pinning controllers will be added to parts of the response network. Without loss of generality, rearrange the order of the nodes in the response network and let the first $l$ nodes be controlled. Then the pinning controlled response network can be described by

$$
\begin{gathered}
\dot{y}^{i}(t)=f\left(y^{i}(t), t\right)+c \sum_{j=1}^{N} a_{i j} h\left(y^{j}(t)\right) \\
-c d_{i}\left(x^{i}(t)+y^{i}(t)\right), \quad i=1,2, \ldots, l, \\
\dot{y}^{i}(t)=f\left(y^{i}(t), t\right)+c \sum_{j=1}^{N} a_{i j} h\left(y^{j}(t)\right), \\
i=l+1, l+2, \ldots, N,
\end{gathered}
$$

where $N>1$ is the network size, $x^{i}(t)=\left(x_{1}^{i}(t), x_{2}^{i}(t), \ldots\right.$, $\left.x_{n}^{i}(t)\right)^{T} \in R^{n}, y^{i}(t)=\left(y_{1}^{i}(t), y_{2}^{i}(t), \ldots, y_{n}^{i}(t)\right)^{T} \in R^{n}$ are the state variables of the $i$-node in the driving network and the response network, respectively, $f: R^{n} \times[0,+\infty) \rightarrow R^{n}$ is a continuous function that describes the local dynamics of the driving networks and the response network, respectively, $c$ is the coupling strength, and $k_{i}(i=1,2, \ldots, l)$ are control gains. $A=\left[a_{i j}\right] \in R^{n \times n}$ denotes the coupling configuration of the network and $a_{i j}$ is defined as follows. If nodes $i$ and $j$ are linked by an edge then $a_{i j}>0$, otherwise $a_{i j}=0$. This ensures that the network is connected, so $A$ is irreducible although $A$ is not necessary symmetric. The nonlinear coupling function is $h\left(x^{i}(t)\right)=\left(g\left(x_{1}^{i}(t)\right), g\left(x_{2}^{i}(t)\right), \ldots, g\left(x_{n}^{i}(t)\right)\right)^{T}, i=$ $1,2, \ldots, N$. 


\section{Model Description}

In this section, we write down some definitions, notations, and lemmas that will be used throughout this paper.

Definition 1 (see [32]). If

$$
\begin{gathered}
\lim _{t \rightarrow \infty}\left\|x^{i}(t)-x^{j}(t)\right\|=0, \quad \lim _{t \rightarrow \infty}\left\|y^{i}(t)-y^{j}(t)\right\|=0, \\
\lim _{t \rightarrow \infty}\left\|x^{i}(t)+y^{i}(t)\right\|=0, \quad i, j=1,2, \ldots, N,
\end{gathered}
$$

then the driving network (3) and the response network (4) are said to achieve hybrid synchronization.

Definition 2 (see [36]). A nonlinear coupling function $g(\cdot)$ : $R \rightarrow R$ is said to belong to the acceptable nonlinear coupling function class, denoted by $h \in \operatorname{NCF}(\gamma, \beta)$, if there exist two nonnegative scalers $\gamma$ and $\beta$ such that $g(x)-\gamma x$ satisfies the following Lipschitz condition:

$$
\left|g\left(x_{1}\right)-g\left(x_{2}\right)-\gamma\left(x_{1}-x_{2}\right)\right| \leq \beta\left|x_{1}-x_{2}\right|
$$

Holds, for any $x_{1}, x_{2} \in R$.

Remark 3. Let $r(x)=g(x)-\gamma x$. Then the above condition (6) can be changed as

$$
\left|r\left(x_{1}\right)-r\left(x_{2}\right)\right| \leq \beta\left|x_{1}-x_{2}\right|
$$

and if $g(\cdot)$ is an odd function, then we have

$$
\left|r\left(x_{1}\right)+r\left(x_{2}\right)\right| \leq \beta\left|x_{1}+x_{2}\right| \text {. }
$$

Definition 4 (see [37]). $A=\left[a_{i j}\right] \in R^{N \times N}$ is said to belong to class $A_{1}$, denoted by $A \in A_{1}$, if

(1) $a_{i j} \geq 0(i \neq j)$, and $a_{i i}=-\sum_{j=1, j \neq i}^{N} a_{i j}$, for $i=$ $1,2, \ldots, N$,

(2) $A$ is irreducible.

If $A \in A_{1}$ is symmetrical, then we say that $A$ belongs to $A_{2}$, denoted by $A \in A_{2}$.

Lemma 5 (see [36]). Assume that $A \in A_{1}$. Then

(1) $\operatorname{rank}(A)=N-1$, that is, 0 is an eigenvalue of $A$ of multiplicity 1, and all nonzero eigenvalues of $A$ have negative real parts;

(2) $\mathbf{1}=(1,1, \ldots, 1)^{T}$ is the right eigenvector of $A$ corresponding to the eigenvalue 0 of multiplicity 1 , that is, $A \cdot \mathbf{1}=0$;

(3) let $\xi=\left(\xi^{1}, \xi^{2}, \ldots, \xi^{N}\right)^{T}$ be the left eigenvector of $A$ corresponding to the eigenvalue 0 , that is, $\xi^{T} A=0$. Then, $\xi^{i}>0, i=1,2, \ldots, N$, and its multiplicity is 1 . In the following, we will always assume that $\sum_{i=1}^{N} \xi^{i}=1$.

Definition 6 (see [36]). Denote $\Xi=\operatorname{diag}\left\{\xi^{1}, \xi^{2}, \ldots, \xi^{N}\right\}, I_{N}=$ $\operatorname{diag}\{1,1, \ldots, 1\}$, and define

$$
U \equiv\left[u_{i j}\right]=\Xi-\xi \xi^{T}, \quad Q=I_{N}-\frac{1}{N} \mathbf{1} \cdot \mathbf{1}^{T} .
$$

Obviously, $U, Q \in A_{2}$. Moreover, it is easy to check that if $A \in A_{1}$ then $A Q=A$.

Lemma 7 (see [37]). If $A=\left(a_{i j}\right) \in A_{2}$, then for any two vectors $u=\left[u_{1}, u_{2}, \ldots, u_{n}\right]^{T}$ and $v=\left[v_{1}, v_{2}, \ldots, v_{n}\right]^{T}$, we have

$$
u^{T} A v=\sum_{i=1}^{n} \sum_{j=1}^{n} u_{i} a_{i j} v_{j}=-\sum_{j>i} a_{i j}\left(u_{i}-u_{j}\right)\left(v_{i}-v_{j}\right)
$$

Hypothesis. Let $P=\operatorname{diag}\left\{p_{1}, p_{2}, \ldots, p_{n}\right\}, p_{i}>0, i=1,2, \ldots$, $n, f(x, t) \in C\left[R^{n} \times[0, \infty), R^{n}\right]$, satisfies

$$
\begin{gathered}
(x-y)^{T} P((f(x, t)-f(y, t))-\Delta(x-y)) \\
\leq-\alpha(x-y)^{T}(x-y),
\end{gathered}
$$

for some $\alpha>0$, all $x, y \in R^{n}$ and $t>0$ where $\Delta=$ $\operatorname{diag}\left\{\delta_{1}, \delta_{2}, \ldots, \delta_{n}\right\}$.

Remark 8 . When $f(x, t)$ is odd with regard to $x$, then we have

$$
\begin{gathered}
(x+y)^{T} P((f(x, t)+f(y, t))-\Delta(x+y)) \\
\leq-\alpha(x+y)^{T}(x+y),
\end{gathered}
$$

for some $\alpha>0$, all $x, y \in R^{n}$, and $t>0$.

\section{Main Result}

In this part, we investigate the hybrid synchronization of a class of nonlinearly coupled complex dynamical networks and obtain some criteria for hybrid synchronization.

Theorem 9. Suppose Hypothesis holds, $A=\left(a_{i j}\right) \in A_{1}, g(\cdot) \in$ $\operatorname{NCF}(\gamma, \beta)$, and $g$ is an odd function, $f(x, t)$ is odd with respect to $x$. If there exist positive scalers $\theta_{k}, \vartheta_{k}(k=1,2, \ldots, n)$ such that the following inequalities

$$
\begin{array}{r}
\delta_{k} U+c \gamma(\Xi A)^{s}+\frac{c \theta_{k}}{2} \Xi A A^{T} \Xi+\frac{c \beta^{2}}{2 \theta_{k}} Q \leq 0, \\
c \gamma B^{s}+\frac{c}{2}\left(\vartheta_{k} B B^{T}+\frac{\beta^{2}}{\vartheta_{k}} I_{N}\right)+\vartheta_{k} I_{N} Q \leq 0, \\
k=1,2, \ldots, n
\end{array}
$$

hold, where $\Xi, U, Q$ are defined as in Definition $6, D=$ $\operatorname{diag}\left\{d_{1}, d_{2}, \ldots, d_{l}, 0, \ldots, 0\right\} \in R^{N \times N}$ and $B=A-D=\left(b_{i j}\right) \epsilon$ $R^{N \times N}$ with $b_{i i}=a_{i i}-d_{i}(i=1,2, \ldots, l)$, otherwise $b_{i j}=a_{i j}$, then the nonlinearly coupled driving network (3) can achieve hybrid synchronization with the response network (4). 
Proof. Denote

$$
\begin{aligned}
& X(t)=\left(x^{1}(t)^{T}, x^{2}(t)^{T}, \ldots, x^{N}(t)^{T}\right)^{T}, \\
& F(X(t))=\left(f\left(x^{1}(t), t\right)^{T}, f\left(x^{2}(t), t\right)^{T}, \ldots,\right. \\
& \left.\quad f\left(x^{N}(t), t\right)^{T}\right)^{T}, \\
& H(X(t))=\left(h\left(x^{1}(t)\right)^{T}, h\left(x^{2}(t)\right)^{T}, \ldots, h\left(x^{N}(t)\right)^{T}\right)^{T} .
\end{aligned}
$$

Then the driving network (3) can be written in a compact form as

$$
X(t)=F(X(t))+c\left(A \otimes I_{n}\right) H(X(t)) .
$$

In order to achieve the hybrid synchronization of two nonlinearly coupled complex networks with different local dynamical systems, we first choose the synchronization state of the drive network to be $s^{\xi}(t)=\sum_{i=1}^{N} \xi^{i} x^{i}(t)$ so that the drive network can be synchronized with $s^{\xi}(t)$. At the same time, we antisynchronize the sates $x^{i}(t)$ of the drive network and the state $y^{i}(t)$ of the response network using pinning control so that (5) is satisfied, that is, the drive and response networks are hybrid synchronized.

Let $e^{i}(t)=x^{i}(t)+y^{i}(t)$. Then the antisynchronization error systems between the driving network (3) and the response network (4) can be written as

$$
\begin{aligned}
\frac{d e^{i}(t)}{d t}= & f\left(x^{i}(t), t\right)+f\left(y^{i}(t), t\right) \\
& +c \sum_{j=1}^{N} b_{i j}\left(h\left(x^{j}(t)\right)+h\left(y^{j}(t)\right)\right) \\
& -c d_{i}\left(h\left(x^{i}(t)\right)+h\left(y^{i}(t)\right)\right), \quad i=1,2, \ldots, l, \\
\frac{d e^{i}(t)}{d t}= & f\left(x^{i}(t), t\right)+f\left(y^{i}(t), t\right) \\
& +c \sum_{j=1}^{N} b_{i j}\left(h\left(x^{j}(t)\right)+h\left(y^{j}(t)\right)\right), \\
& i=l+1, l+2, \ldots, N .
\end{aligned}
$$

Construct a Lyapunov function candidate as

$$
V(t)=V_{1}(t)+V_{2}(t)
$$

where

$$
\begin{aligned}
V_{1}(t) & =\frac{1}{2} \sum_{i=1}^{N} \xi^{i}\left(x^{i}(t)-x^{\xi}(t)\right)^{T} P\left(x^{i}(t)-x^{\xi}(t)\right) \\
& =\frac{1}{2} X(t)^{T}(U \otimes P) X(t), \\
V_{2}(t) & =\frac{1}{2} \sum_{i=1}^{N} e^{i}(t)^{T} P e^{i}(t) .
\end{aligned}
$$

Differentiating the function $V_{1}(t)$ along the drive network (15), we have

$$
\begin{aligned}
\dot{V}_{1}(t)= & X(t)^{T}(U \otimes P)\left(F(X(t))+c\left(A \otimes I_{n}\right) H(X(t))\right) \\
= & X(t)^{T}(U \otimes P)(F(X(t))-(I \otimes \Delta) X(t)) \\
& +X(t)^{T}(U \otimes P) \\
& \times\left((I \otimes \Delta) X(t)+c\left(A \otimes I_{n}\right) H(X(t))\right) \\
= & V_{11}(t)+V_{12}(t) .
\end{aligned}
$$

Because of Hypothesis (11) and Lemma 7, we have

$$
\begin{aligned}
V_{11}(t)= & X(t)^{T}(U \otimes P)(F(X(t))-(I \otimes \Delta) X(t)) \\
= & -\sum_{i>j}^{N} u_{i j}\left(x^{i}(t)-x^{j}(t)\right)^{T} \\
& \times P\left(f\left(x^{i}(t), t\right)-f\left(x^{j}(t), t\right)\right. \\
\leq & \alpha \sum_{i>j}^{N} u_{i j}\left(x^{i}(t)-x^{j}(t)\right)^{T}\left(x^{i}(t)-s^{j}(t)\right) \\
= & -\alpha X(t)^{T} U \otimes I X(t) \\
\leq & -\frac{\alpha}{\max _{k} p_{k}} X(t)^{T}(U \otimes P) X(t) \\
= & -\frac{2 \alpha}{\max _{k} p_{k}} V_{1}(t) .
\end{aligned}
$$

Denote $\tilde{x}_{k}(t)=\left(x_{k}^{1}(t), x_{k}^{2}(t), \ldots, x_{k}^{N}(t)\right)^{T}, \widetilde{h}_{k}\left(\widetilde{x}_{k}(t)\right)=$ $\left(g\left(x_{k}^{1}(t)\right), g\left(x_{k}^{2}(t)\right), \ldots, g\left(x_{k}^{N}(t)\right)\right)^{T}$ for $k=1,2, \ldots, n$ and rewrite $V_{12}(t)$ as follows

$$
\begin{aligned}
V_{12}(t)= & X(t)^{T}(U \otimes P \Delta) X(t)+c X(t)^{T}(U A \otimes P) H(X(t)) \\
= & \sum_{k=1}^{n} p_{k} \delta_{k} \widetilde{x}_{k}(t)^{T} U \widetilde{x}_{k}(t)+c \sum_{k=1}^{n} p_{k} \widetilde{x}_{k}(t)^{T} \Xi A \widetilde{h}_{k}\left(\widetilde{x}_{k}(t)\right) \\
= & \sum_{k=1}^{n} p_{k} \tilde{x}_{k}(t)^{T}\left(\delta_{k} U+c \gamma \Xi A\right) \tilde{x}_{k}(t) \\
& +c \sum_{k=1}^{n} p_{k} \tilde{x}_{k}(t)^{T} \Xi A\left(\widetilde{h}_{k}\left(\tilde{x}_{k}(t)\right)-\gamma \tilde{x}_{k}(t)\right) .
\end{aligned}
$$


Since $U A=\Xi A, A \in A_{1}$, and from the property of $\mathrm{Q}$ in Definition 6 gives

$$
\begin{aligned}
& \tilde{x}_{k}(t)^{T} \Xi A\left(\tilde{h}_{k}\left(\tilde{x}_{k}(t)\right)-\gamma \tilde{x}_{k}(t)\right) \\
& =\tilde{x}_{k}(t)^{T} \Xi A Q\left(\widetilde{h}_{k}\left(\tilde{x}_{k}(t)\right)-\gamma \tilde{x}_{k}(t)\right) \\
& \leq \frac{1}{2}\left(\theta_{k} \tilde{x}_{k}(t)^{T} \Xi A A^{T} \Xi \tilde{x}_{k}(t)+\frac{1}{\theta_{k}}\left(\widetilde{h}_{k}\left(\tilde{x}_{k}(t)\right)-\gamma \tilde{x}_{k}(t)\right)^{T}\right. \\
& \left.\times Q\left(\widetilde{h}_{k}\left(\tilde{x}_{k}(t)\right)-\gamma \tilde{x}_{k}(t)\right)\right) .
\end{aligned}
$$

By Lemma 7 and $g \in \operatorname{NCF}(\gamma, \beta)$, we have

$$
\begin{gathered}
\left(\widetilde{h}_{k}\left(\tilde{x}_{k}(t)\right)-\gamma \widetilde{x}_{k}(t)\right)^{T} Q\left(\widetilde{h}_{k}\left(\tilde{x}_{k}(t)\right)-\gamma \tilde{x}_{k}(t)\right) \\
\quad=-\sum_{i>i} Q_{i j}\left(g\left(x_{k}^{j}(t)\right)-\gamma x_{k}^{j}(t)-g\left(x_{k}^{i}(t)\right)+\gamma x_{k}^{i}(t)\right)^{2} \\
\quad \leq-\beta^{2} \sum_{i>j} Q_{i j}\left(x_{k}^{j}(t)-x_{k}^{i}(t)\right)^{2}=\beta^{2} \tilde{x}_{k}(t)^{T} Q \tilde{x}_{k}(t) .
\end{gathered}
$$

Combining inequalities (22) and (23), we obtain

$$
\begin{aligned}
& c \sum_{k=1}^{n} p_{k} \tilde{x}_{k}(t)^{T} \Xi A\left(\widetilde{h}_{k}\left(\tilde{x}_{k}(t)\right)-\gamma \tilde{x}_{k}(t)\right) \\
& \quad \leq \frac{c}{2} \sum_{k=1}^{n} p_{k} \tilde{x}_{k}(t)^{T}\left(\theta_{k} \Xi A A^{T} \Xi+\frac{\beta^{2}}{\theta_{k}} Q\right) \tilde{x}_{k}(t) .
\end{aligned}
$$

Substituting inequalities (24) into (21), we get

$$
\begin{aligned}
V_{12}(t) \leq & \sum_{k=1}^{n} p_{k} \tilde{x}_{k}(t)^{T} \\
& \times\left(\delta_{k} U+c \gamma \Xi A+\frac{c}{2} \theta_{k} \Xi A A^{T} \Xi+\frac{c}{2} \frac{\beta^{2}}{\theta_{k}} Q\right) \\
& \times \tilde{x}_{k}(t) \leq 0,
\end{aligned}
$$

which means that

$$
\begin{array}{r}
\frac{d V_{1}(t)}{d t} \leq-\frac{2 \alpha}{\max _{k} p_{k}} V_{1}(t) \triangleq-\phi V_{1}(t), \\
\left(\phi=-\frac{2 \alpha}{\max _{k} p_{k}}<0\right) .
\end{array}
$$

Thus, $V_{1}(t)=o\left(e^{-\phi t}\right)$. From the definition of $V_{1}(t)$, we have $\left(x^{i}(t)-x^{\xi}(t)\right)^{T}\left(x^{i}(t)-x^{\xi}(t)\right)=o\left(e^{-\phi t}\right)$ for $i=1,2, \ldots, N$, which means $x^{i}(t)-x^{\xi}(t)$ converges to zero exponentially. Therefore, $x^{i}(t)$ can be synchronized with $x^{\xi}(t)$ globally and exponentially.
Evaluating the time derivative of $V_{2}(t)$ along the trajectory of (16), we get

$$
\begin{aligned}
\frac{d V_{2}(t)}{d t}=\sum_{i=1}^{N} e^{i}(t)^{T} P\left[f\left(x^{i}(t), t\right)+f\left(y^{i}(t), t\right)\right. & \left.\begin{array}{c}
{ }^{T} \sum_{j=1}^{N} a_{i j}\left(h\left(x^{i}(t)\right)+h\left(y^{i}(t)\right)\right) \\
-c d_{i}\left(h\left(x^{j}(t)\right)+h\left(y^{j}(t)\right)\right)
\end{array}\right] \\
=\sum_{i=1}^{N} e^{i}(t)^{T} P\left[f\left(x^{i}(t), t\right)+f\left(y^{i}(t), t\right)\right. & \left.-\Delta\left(x^{i}(t)+y^{i}(t)\right)\right] \\
& +\sum_{i=1}^{N} e^{i}(t)^{T} P \Delta e^{i}(t) \\
& +c \sum_{i=1}^{N} e^{i}(t)^{T} P \sum_{j=1}^{N} b_{i j}\left(h\left(x^{j}(t)\right)+h\left(y^{j}(t)\right)\right) \\
= & V_{21}(t)+V_{22}(t)+V_{23}(t) .
\end{aligned}
$$

By Hypothesis and because $f$ is odd respect to $x$, we obtain

$$
\begin{gathered}
V_{21}(t)=\sum_{i=1}^{N} e^{i}(t)^{T} P\left[f\left(x^{i}(t), t\right)+f\left(y^{i}(t), t\right)\right. \\
\left.-\Delta\left(x^{i}(t)+y^{i}(t)\right)\right] \\
\leq-\alpha \sum_{i=1}^{N} e^{i}(t)^{T} e^{i}(t) .
\end{gathered}
$$

Let $\widetilde{e}_{k}(t)=\tilde{x}_{k}(t)+\tilde{y}_{k}(t)$. Since $g \in \operatorname{NCF}(\gamma, \beta), h_{k}\left(\tilde{x}_{k}(t)\right)$ can be as $h_{k}\left(\tilde{x}_{k}(t)\right)=\gamma \tilde{x}_{k}(t)+\tilde{r}_{k}\left(\tilde{x}_{k}(t)\right)$ where $\tilde{r}_{k}\left(\tilde{x}_{k}(t)\right)=$ $\left(r\left(x_{k}^{1}(t)\right), r\left(x_{k}^{2}(t)\right), \ldots, r\left(x_{k}^{N}(t)\right)\right)^{T}, k=1,2, \ldots, n$, we have

$$
\begin{aligned}
V_{22}(t)= & c \sum_{i=1}^{N} e^{i}(t)^{T} P \sum_{j=1}^{N} b_{i j}\left(h\left(x^{j}(t)\right)+h\left(y^{j}(t)\right)\right) \\
= & c \sum_{k=1}^{n} p_{k} \widetilde{e}_{k}(t)^{T} B\left(h_{k}\left(\tilde{x}_{k}(t)\right)+h_{k}\left(\widetilde{y}_{k}(t)\right)\right) \\
= & c \gamma \sum_{k=1}^{n} p_{k} \widetilde{e}_{k}(t)^{T} B^{s} \widetilde{e}_{k}(t) \\
& +c \sum_{k=1}^{n} p_{k} \widetilde{e}_{k}(t)^{T} B\left(\widetilde{r}_{k}\left(\tilde{x}_{k}(t)\right)+\widetilde{r}_{k}\left(\tilde{y}_{k}(t)\right)\right) .
\end{aligned}
$$


Since $g$ is odd and there exists $\vartheta_{k}>0$ for $1 \leq k \leq n$, we have

$$
\begin{aligned}
\widetilde{e}_{k}(t)^{T} B\left(\widetilde{r}_{k}\left(\widetilde{x}_{k}(t)\right)+\widetilde{r}_{k}\left(\widetilde{y}_{k}(t)\right)\right) \\
\leq \frac{1}{2}\left(\vartheta_{k} \widetilde{e}_{k}(t)^{T} B B^{T} \widetilde{e}_{k}(t)+\frac{1}{\vartheta_{k}}\left(\widetilde{r}_{k}\left(\widetilde{x}_{k}(t)\right)+\widetilde{r}_{k}\left(\widetilde{y}_{k}(t)\right)\right)^{T}\right. \\
\left.\quad \times\left(\widetilde{r}_{k}\left(\widetilde{x}_{k}(t)\right)+\widetilde{r}_{k}\left(\widetilde{y}_{k}(t)\right)\right)\right) \\
=\frac{1}{2}\left(\vartheta_{k} \widetilde{e}_{k}(t)^{T} B B^{T} \widetilde{e}_{k}(t)\right. \\
\left.\quad+\frac{1}{\vartheta_{k}} \sum_{s=1}^{N}\left(r\left(x_{k}^{s}(t)\right)+r\left(y_{k}^{s}(t)\right)\right)^{2}\right) \\
\leq \frac{1}{2}\left(\vartheta_{k} \widetilde{e}_{k}(t)^{T} B B^{T} \widetilde{e}_{k}(t)+\frac{\beta^{2}}{\vartheta_{k}} \sum_{s=1}^{N}\left(x_{k}^{s}(t)+y_{k}^{s}(t)\right)^{2}\right) \\
=\frac{1}{2} \widetilde{e}_{k}(t)^{T}\left(\vartheta_{k} B B^{T}+\frac{\beta^{2}}{\vartheta_{k}} I_{N}\right) \widetilde{e}_{k}(t) .
\end{aligned}
$$

Substituting inequality (30) into (29), we get

$$
V_{22}(t) \leq \sum_{k=1}^{n} p_{k} \widetilde{e}_{k}(t)^{T}\left\{c \gamma B^{s}+\frac{c}{2}\left[\vartheta_{k} B B^{T}+\frac{\beta^{2}}{\vartheta_{k}} I_{N}\right]\right\} \widetilde{e}_{k}(t) .
$$

On the other hand, $V_{23}(t)$ can be directly written as

$$
V_{23}(t)=\sum_{k=1}^{n} p_{k} \widetilde{e}_{k}(t)^{T} \delta_{k} \widetilde{e}_{k}(t) .
$$

Finally, substituting (28), (31), and (32) into (27), we get

$$
\begin{aligned}
\frac{d V_{2}(t)}{d t} \leq & -\alpha \sum_{i=1}^{N} e^{i}(t)^{T} e^{i}(t)+\sum_{k=1}^{n} p_{k} \widetilde{e}_{k}(t)^{T} \\
& \times\left\{c \gamma B^{s}+\frac{c}{2}\left(\vartheta_{k} B B^{T}+\frac{\beta^{2}}{\vartheta_{k}} I_{N}\right)+\delta_{k} I_{N}\right\} \widetilde{e}_{k}(t) .
\end{aligned}
$$

It now implies that

$$
\frac{d V_{2}(t)}{d t} \leq-\alpha \sum_{i=1}^{N} e^{i}(t)^{T} e^{i}(t)
$$

and $V_{2}(t)=o\left(e^{-\alpha t}\right)$ follows. Thus, $e^{i}(t) \rightarrow 0(t \rightarrow \infty)$ for $i=1,2, \ldots, N$. This implies that

$$
\lim _{t \rightarrow+\infty}\left\|x^{i}(t)+y^{i}(t)\right\|=0, \quad i=1,2, \ldots, N .
$$

The proof of the theorem is complete.

From the proof, one can see that the difficulty in investigating the hybrid synchronization with an asymmetrical coupling matrix $A$ lies in how to deal with the terms $\widetilde{x}_{k}(t)^{T} \Xi A \widetilde{h}_{k}\left(\widetilde{x}_{k}(t)\right)$ of $(22)$ and $\widetilde{e}_{k}(t)^{T} B\left(h_{k}\left(\widetilde{x}_{k}(t)\right)+h_{k}\left(\widetilde{y}_{k}(t)\right)\right)$ of (31), where $\Xi A$ and $B$ are asymmetrical. If $A$ is symmetric, then $\Xi A$ and $B$ are symmetric. In the case, by Lemma 7, we have

$$
\begin{aligned}
& c \sum_{k=1}^{n} p_{k} \widetilde{x}_{k}(t)^{T} A \widetilde{h}_{k}\left(\widetilde{x}_{k}(t)\right) \\
& =c \sum_{k=1}^{n} p_{k} \sum_{i>j} a_{i j}\left(x_{k}^{i}-x_{k}^{j}\right)\left(g\left(x_{k}^{i}\right)-g\left(x_{k}^{j}\right)\right) \\
& \leqslant-c \underline{\gamma} \sum_{k=1}^{n} p_{k} \sum_{i>j} a_{i j}\left(x_{k}^{i}-x_{k}^{j}\right)^{2} \\
& =c \underline{\gamma} \sum_{k=1}^{n} p_{k} \tilde{x}_{k}(t)^{T} A \tilde{x}_{k}(t),
\end{aligned}
$$

where $\underline{\gamma}$ is defined as $\underline{\underline{\gamma}}=\inf _{w_{1} \neq w_{2}}\left(g\left(w_{1}\right)-g\left(w_{2}\right)\right) /\left(w_{1}-w_{2}\right)>$ 0 , for $\bar{w}_{1}, w_{2} \in R$.

Therefore

$$
V_{12}(t) \leq \sum_{k=1}^{n} p_{k} \widetilde{x}_{k}(t)^{T}\left(\delta_{k} U+\frac{c}{N} \underline{\gamma} A\right) \widetilde{x}_{k}(t)
$$

on the other hand, let $\widetilde{e}_{k}^{i}(t)=x_{k}^{i}(t)+y_{k}^{i}(t)$, from (29), we get

$$
\begin{aligned}
V_{22}(t)= & c \sum_{k=1}^{n} p_{k} \widetilde{e}_{k}(t)^{T} B\left(\widetilde{h}\left(\widetilde{x}_{k}(t)\right)+\widetilde{h}\left(\widetilde{y}_{k}(t)\right)\right) \\
= & c \sum_{k=1}^{n} p_{k} \sum_{i>j} b_{i j}\left(\widetilde{e}_{k}^{i}(t)-\widetilde{e}_{k}^{j}(t)\right) \\
& \times\left(g\left(x_{k}^{i}(t)\right)+g\left(y_{k}^{i}(t)\right)-g\left(x_{k}^{j}(t)\right)-g\left(y_{k}^{j}(t)\right)\right) \\
= & c \underline{\gamma} \sum_{k=1}^{n} p_{k} \sum_{i>j} b_{i j}\left(\widetilde{e}_{k}^{i}(t)-\widetilde{e}_{k}^{j}(t)\right)^{2} \\
= & c \underline{\gamma} \sum_{k=1}^{n} p_{k} \widetilde{e}_{k}(t)^{T} B \widetilde{e}_{k}(t) .
\end{aligned}
$$

Notice (36), (37), and (38), we obtain the following corollaries.

Corollary 10. Suppose $f(\cdot)$ satisfy Hypothesis. $A=\left(a_{i j}\right) \in A_{2}$, $g(\cdot) \in N C F(\gamma, \beta)$, and $g$ is odd function, $f(x, t)$ is odd with regard to $x$, if the following inequalities

$$
\begin{aligned}
& \delta_{k} U+\frac{c}{N} \underline{\gamma} A \leq 0, \\
& \underline{\gamma} B+\delta_{k} I_{N} \leq 0,
\end{aligned}
$$

hold, then the nonlinearly coupled driving network (3) can hybrid synchronized to the response network(4). 
If $g \in N C F(\gamma, 0)$, then the driving network (3) becomes linear-coupling systems

$$
\dot{x}^{i}(t)=f\left(x^{i}(t), t\right)+c \sum_{j=1}^{N} a_{i j} x^{j}(t), \quad i=1,2, \ldots, N,
$$

respectively, the pinning controlled response network (4) can be rewritten as

$$
\begin{gathered}
\dot{y}^{i}(t)=f\left(y^{i}(t), t\right)+c \sum_{j=1}^{N} a_{i j} y^{j}(t)-c d_{i}\left(x^{i}(t)+y^{i}(t)\right), \\
i=1,2, \ldots, l, \\
\dot{y}^{i}(t)=f\left(y^{i}(t), t\right)+c \sum_{j=1}^{N} a_{i j} y^{j}(t), \quad i=l+1, l+2, \ldots, N .
\end{gathered}
$$

As a direct consequence of Theorem 9, we obtain the following result.

Corollary 11. Suppose Hypothesis holds. $A=\left(a_{i j}\right) \in A_{1}$, $g(\cdot) \in N C F(\gamma, 0)$, and $g$ is odd function, $f(x, t)$ is odd with regard to $x$, if the following inequalities

$$
\begin{aligned}
& \delta_{k} U+c \gamma(\Xi A)^{s} \leq 0, \\
& c \gamma B^{s}+\delta_{k} I_{N} \leq 0
\end{aligned}
$$

hold, then the linearly coupled driving network (40) can hybrid synchronized to the response network (41).

The case has been discussed in [30]. Therefore, one can see the main result of the paper as a nontrivial extension of the Theorem 1 of [30].

Remark 12. By using the Lyapunov method combined with some other technique, the hybrid synchronization criterion of the pinning-controlled dynamical networks has been obtained. It means that the outer antisynchronization between system (3) and system (4) while the inner synchronization in them. Our result displays that pinning control is effective for the hybrid synchronization of complex dynamical network. Pinning impulsive control strategy has been used effectively for the synchronization of stochastic dynamical networks with nonlinear coupling in $[33,35]$. Our pinning control strategy for the hybrid synchronization of two nonlinearly coupled complex networks in this paper differs from them.

\section{Numerical Simulation}

In this section, we given numerical simulation to verify the theorem given in the previous section. In order to verify our results, we consider the driving complex network [32] as

$$
\dot{x}^{i}(t)=f\left(x^{i}(t), t\right)+c \sum_{j=1}^{5} a_{i j} h\left(x^{j}(t)\right), \quad i=1,2, \ldots, 5
$$

and the response networks [32] as

$$
\begin{aligned}
\dot{y}^{i}(t)= & f\left(y^{i}(t), t\right)+\sum_{j=1}^{5} a_{i j} h\left(y^{j}(t)\right) \\
& -c d_{i}\left(x^{i}(t)+y^{i}(t)\right), \quad i=1,2, \\
\dot{y}^{i}(t)= & f\left(y^{i}(t), t\right)+\sum_{j=1}^{5} a_{i j} h\left(y^{j}(t)\right), \quad i=3,4,5,
\end{aligned}
$$

where

$$
\begin{gathered}
f\left(x^{i}(t), t\right)=-S x^{i}(t)+T w\left(x^{i}(t)\right), \\
S=\left[\begin{array}{ccc}
3.2 & -10 & 0 \\
-1 & 1 & -1 \\
0 & 14.87 & 0
\end{array}\right], \quad T=\left[\begin{array}{ccc}
5.9 & 0 & 0 \\
0 & 0 & 0 \\
0 & 0 & 0
\end{array}\right],
\end{gathered}
$$

and $w(x)=(|x+1|-|x-1|) / 2$.

Let $P=I_{3}, \Delta=\delta I_{3}$, if there exists a positive-definite diagonal matrix $\Sigma=\operatorname{diag}\left\{\sigma_{1}, \ldots, \sigma_{n}\right\}$, such that

$$
\left[\begin{array}{cc}
2 P(S+\Delta)-\Sigma & -P T \\
-T^{T} P & \Sigma
\end{array}\right]>0
$$

then $f\left(x^{i}(t), t\right)$ satisfies the condition (11) [38] in Hypothesis. Using Matlab LMI Control Toolbox, we get $\delta=9.0637$.

Example 13. As for the asymmetrical coupling matrix $A$, we use

$$
A=\left[\begin{array}{ccccc}
-0.794 & 0.794 & 0 & 0 & 0 \\
0 & -0.84 & 0.311 & 0 & 0.529 \\
0.166 & 0 & -0.166 & 0 & 0 \\
0.602 & 0 & 0.263 & -0.865 & 0 \\
0 & 0 & 0 & 0.654 & -0.654
\end{array}\right],
$$

whose left eigenvector corresponding to eigenvalue 0 is $\xi=$ $(0.3157,0.2984,0.8483,0.1825,0.2414)^{T}$. It is clear that $\xi^{T} L=$ 0 . Let the nonlinear function $g(x(t))=5 x(t)+\sin (x(t))$, so $g(\cdot) \in \operatorname{NCF}(5,1)(\gamma=5, \beta=1)$.

The initial values are chosen as

$$
\begin{aligned}
& x^{1}(0)=(-0.4946,-4.1618,-2.7102)^{T}, \\
& x^{2}(0)=(4.1334,-3.4762,3.2582)^{T}, \\
& x^{3}(0)=(0.3834,4.9613,-4.2182)^{T}, \\
& x^{4}(0)=(-0.5732,-3.9335,4.619)^{T}, \\
& x^{5}(0)=(-4.9537,2.7491,3.173)^{T}, \\
& y^{1}(0)=(-3.7009,0.6882,-0.3061)^{T}, \\
& y^{2}(0)=(-4.881,-1.6288,-3.3782)^{T}, \\
& y^{3}(0)=(2.9428,-1.8878,0.2853)^{T}, \\
& y^{4}(0)=(-3.3435,1.0198,-2.3703)^{T}, \\
& y^{5}(0)=(1.5408,1.8921,2.4815)^{T},
\end{aligned}
$$




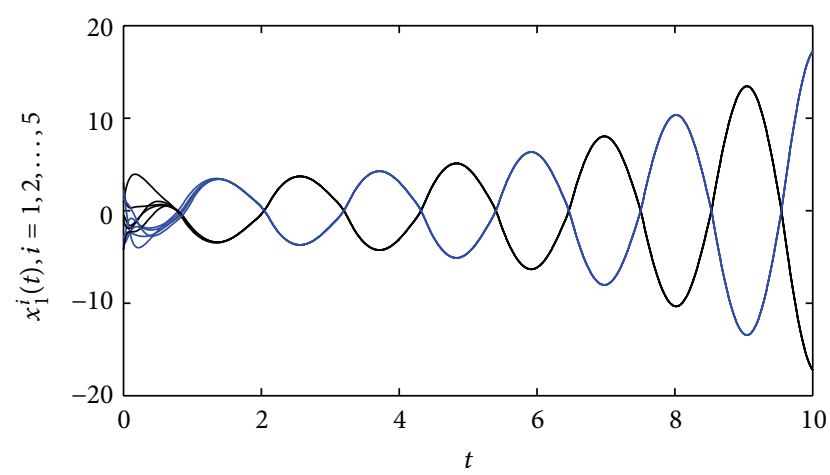

(a)

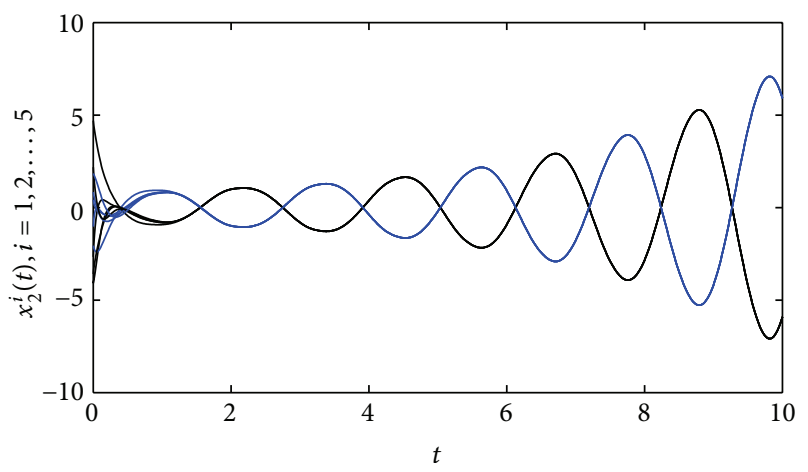

(b)

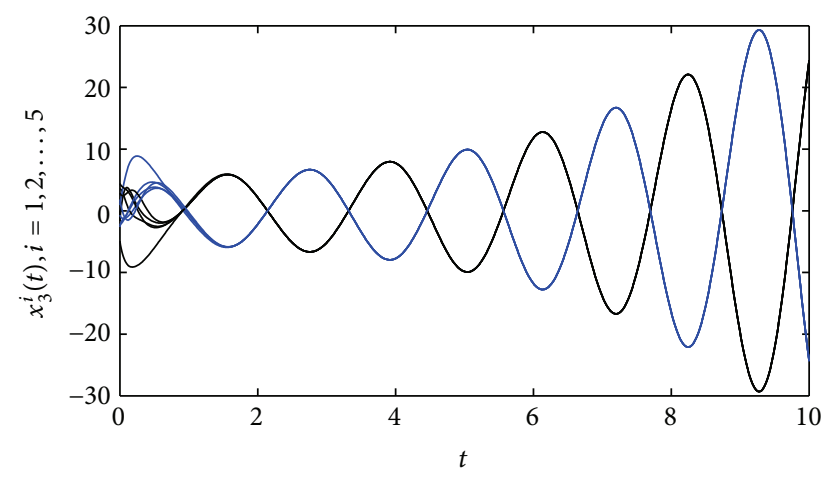

(c)

FIGURE 1: The trajectories evolution of the state variable, (a) $x_{1}^{i}(t)$, $y_{1}^{i}(t),(\mathrm{b}) x_{2}^{i}(t), y_{2}^{i}(t)$, and (c) $x_{3}^{i}(t), y_{3}^{i}(t)(i=1,2, \ldots, 5)$.

and the errors between nodes are defined by

$$
\begin{aligned}
& E_{x}=\sum_{i>j}\left\|x^{i}(t)-x^{j}(t)\right\|, \\
& E_{y}=\sum_{i>j}\left\|y^{i}(t)-y^{j}(t)\right\|, \\
& E_{x y}=\sum_{i=1}^{5} \sum_{j=1}^{5}\left\|x^{i}(t)+y^{j}(t)\right\|,
\end{aligned}
$$

as measures of hybrid synchronization.

Let $c=11.868, \theta_{k}=2.309, \vartheta_{k}=0.965,(k=1,2,3)$, and $d_{1}=d_{2}=1.76, d_{3}=d_{4}=d_{5}=0$, the calculations show

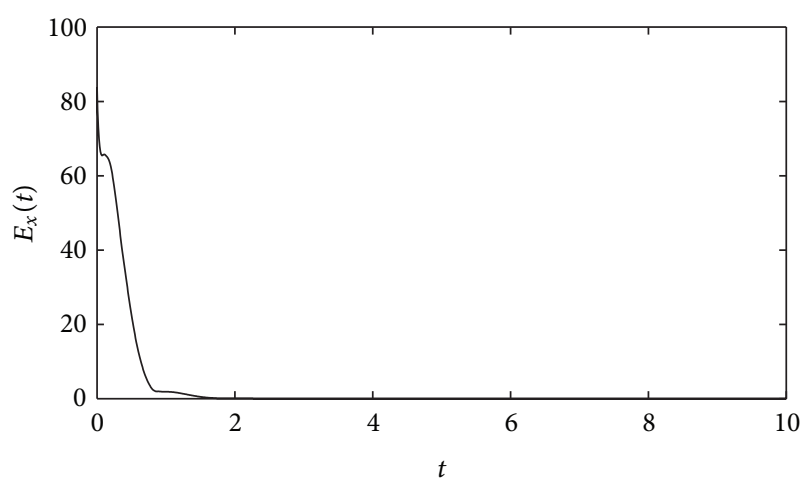

(a)

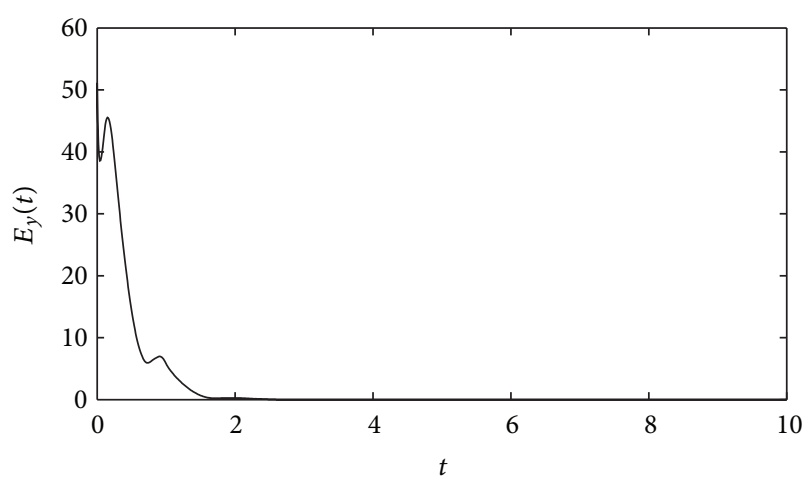

(b)

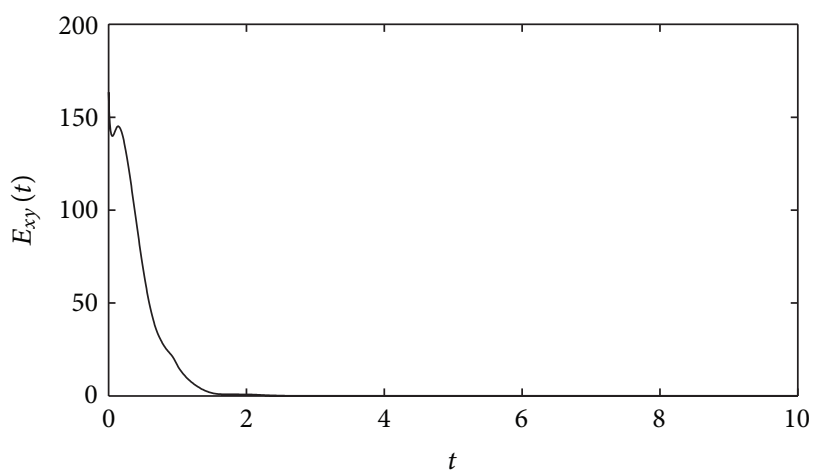

(c)

FIgURE 2: (a) The synchronization error evolution of the driver networks $E_{x}(t)$; (b) The synchronization error evolution of the response networks $E_{y}(t)$; (c) The anti-synchronization error evolution between the driver networks and the response networks $E_{x y}(t)$ with the pinning control.

that eigenvalues of matrix $\delta_{k} U+c \gamma(\Xi A)^{s}+\left(c \theta_{k} / 2\right) \Xi A A^{T} \Xi+$ $\left(c \beta^{2} / 2 \theta_{k}\right) Q$ are $-16.3119,-0.1990,-7.8756,-3.8328$, and -5.5047 , and $c \gamma B^{s}+(c / 2)\left(\vartheta_{k} B B^{T}+\left(\beta^{2} / \vartheta_{k}\right) I_{N}\right)+\delta_{k} I_{N}$ are $-0.0823,-21.76,-45.804,-103.63$, and -77.347 .

The trajectories evolution of the pinning control gains are shown in Figure 1. The evolution of synchronization error of the driver and the response system in Figures 2(a) and 2(b), respectively. The evolution of synchronization error of between the driver and the response system Figure 2(c). 


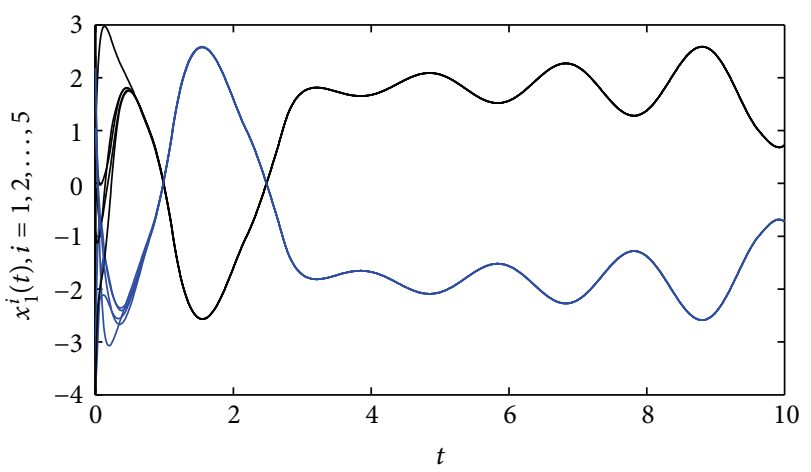

(a)

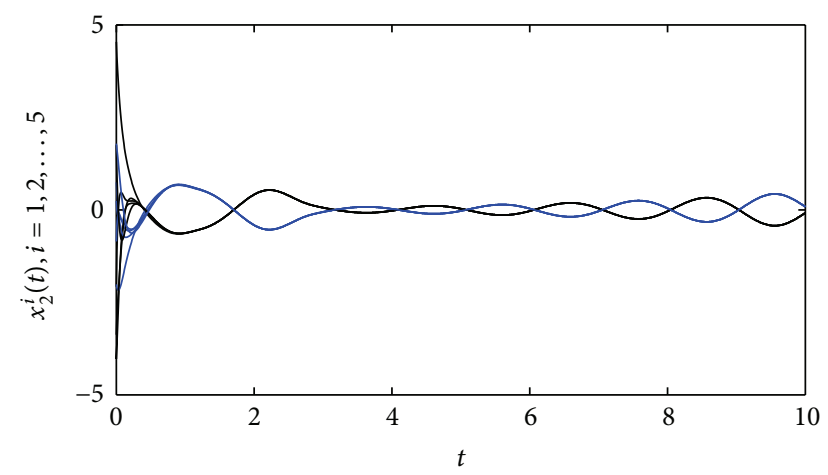

(b)

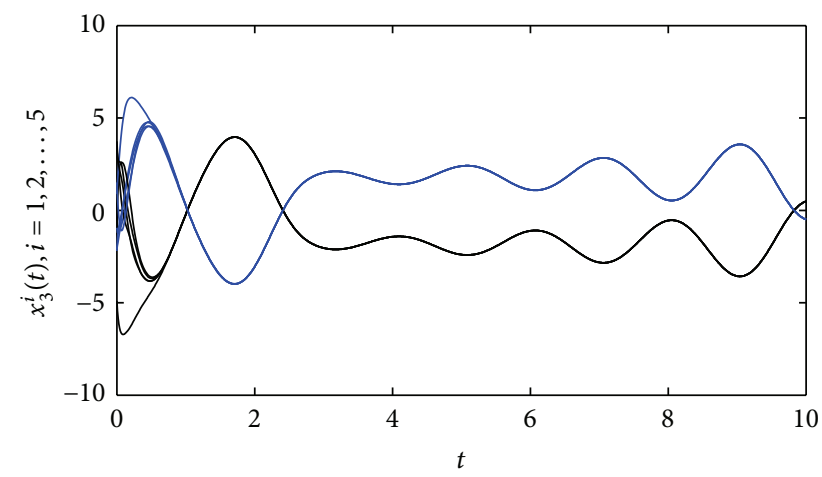

(c)

FIgURE 3: The trajectories evolution of the state variable, (a) $x_{1}^{i}(t)$, $y_{1}^{i}(t),(\mathrm{b}) x_{2}^{i}(t), y_{2}^{i}(t)$, and (c) $x_{3}^{i}(t), y_{3}^{i}(t)(i=1,2, \ldots, 5)$.

Example 14. As for the symmetrical coupling matrix $A$, we use

$$
A=\left[\begin{array}{ccccc}
-2.407 & 0.792 & 0.959 & 0 & 0.656 \\
0.792 & -0.792 & 0 & 0 & 0 \\
0.959 & 0 & -2.572 & 0.934 & 0.679 \\
0 & 0 & 0.934 & -0.934 & 0 \\
0.656 & 0 & 0.679 & 0 & -1.335
\end{array}\right]
$$

Let $c=9.182, d_{1}=d_{1}=1.76$, and $d_{3}=d_{4}=d_{5}=0$, the calculations show that eigenvalues of matrix $\delta_{k} U+(c / N) \gamma A$ are $-30.4559,-18.9805,-15.2688,-9.3311$, and -0.1760 , and $c \gamma B+\delta_{k} I_{N}$ are: $-59.0165,-36.2877,-28.4531,-14.4174$, and $-\overline{0} .2446$.

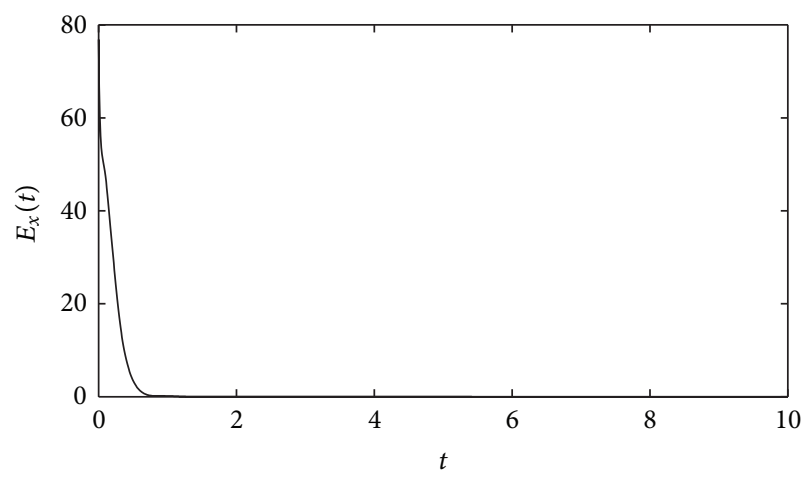

(a)

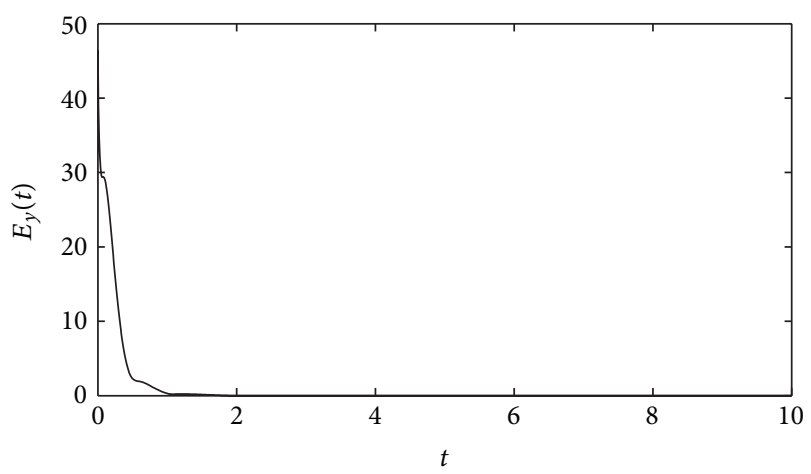

(b)

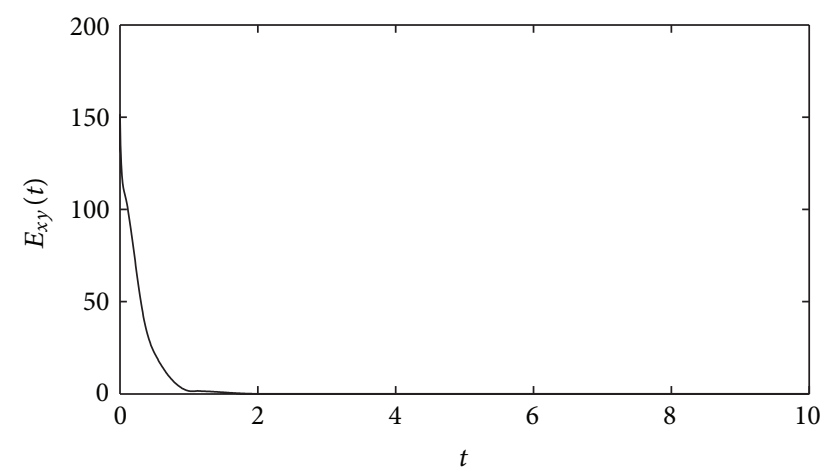

(c)

FIgURE 4: (a) The synchronization error evolution of the driver networks $E_{x}(t)$. (b) The synchronization error evolution of the response networks $E_{y}(t)$. (c) The antisynchronization error evolution between the driver networks and the response networks $E_{x y}(t)$ with the pinning control.

The initial values are chosen as above. The trajectories evolution of the pinning control gains are shown in Figure 3. The evolution of synchronization error of the driver and the response system in Figures 4(a) and 4(b), respectively. The evolution of synchronization error of between the driver and the response system in Figure 4(c).

\section{Conclusions and Future Directions}

In this paper, we considered the hybrid synchronization of two nonlinearly coupled complex dynamical networks with 
asymmetrical coupling matrices under the pinning control scheme. By placing a small number of feedback controllers on some nodes, we obtained some criteria for the hybrid synchronization of such dynamical networks based on Lyapunov Stability Theory. It is shown that under certain conditions, two nonlinearly coupled complex networks can achieve an intriguing hybrid synchronization: the outer antisynchronization between the driving network and the response network and the inner complete synchronization between the driving network and the response network, respectively. Numerical examples were also provided to demonstrate the effectiveness of the theoretical result.

Because the random phenomenon appears frequently in dynamical complex network, in our further efforts, this feature should be taken into account in order to solve problems more practically. More precisely, our future works may be extended to the consensus problem of the multiagent systems with stochastic disturbance, the synchronization issue of the dynamical complex networks with stochastic disturbance, and so on. On the other side, control methods would not be limited to the feedback control any more. For example, in real world, the states of nodes in the networks often suffer from instantaneous perturbations or abrupt changes at certain instants, such as switching phenomena, frequency change, or sudden noise. In order to investigate the synchronization matters for this situation, suitable pinning impulsive controllers could be applied to deal with the impulsive-coupled dynamical networks. Moreover, couplings or communications between nodes in this paper are considered to be continuous. However, there are other modes of the information exchanging among the nodes, such as impulsive communication and intermittent communication. The studies on these problems will be also interesting and meaningful in the further research work.

\section{Acknowledgments}

This work was supported by the National Science Foundation of China under Grant no. 61273220, the Guangdong Education University Industry Cooperation Project (2009B090300355), and the Shenzhen Basic Research Project (JC201006010743A, JCYJ20120613105730482). The authors are very grateful to the reviewers and editors for their valuable comments on this paper.

\section{References}

[1] D. J. Watts and S. H. Strogatz, "Collective dynamics of 'smallworld9 networks," Nature, vol. 393, no. 6684, pp. 440-442, 1998.

[2] S. H. Strogatz, "Exploring complex networks," Nature, vol. 410, no. 6825 , pp. 268-276, 2001.

[3] M. E. J. Newman, "The structure and function of complex networks," SIAM Review, vol. 45, no. 2, pp. 167-256, 2003.

[4] X. F. Wang and G. Chen, "Synchronization in scale-free dynamical networks: robustness and fragility," IEEE Transactions on Circuits and Systems. I. Fundamental Theory and Applications, vol. 49, no. 1, pp. 54-62, 2002.
[5] A. Zheleznyak and L. O. Chua, "Coexistence of low- and highdimensional spatiotemporal chaos in a chain of dissipativelycoupled. Chuas circuits," International Journal of Bifurcation and Chaos, vol. 4, pp. 639-672, 1994.

[6] V. Perez-Munuzuri, V. Perez-Villar, and L. O. Chua, "Autowaves for image processing on a two-dimensional CNN array of excitable nonlinear circuits: flat and wrinkled labyrinths," IEEE Transactions on Circuits and Systems I, vol. 40, no. 3, pp. 174-181, 1993.

[7] C. W. Wu and L. O. Chua, "Synchronization in an array of linearly coupled dynamical systems," IEEE Transactions on Circuits and Systems. I. Fundamental Theory and Applications, vol. 42, no. 8, pp. 430-447, 1995.

[8] J. Cao, P. Li, and W. Wang, "Global synchronization in arrays of delayed neural networks with constant and delayed coupling," Physics Letters A, vol. 353, no. 4, pp. 318-325, 2006.

[9] W. Yu, J. Cao, and J. Lü, "Global synchronization of linearly hybrid coupled networks with time-varying delay," SIAM Journal on Applied Dynamical Systems, vol. 7, no. 1, pp. 108-133, 2008.

[10] J. Lü and G. Chen, "A time-varying complex dynamical network model and its controlled synchronization criteria," IEEE Transactions on Automatic Control, vol. 50, no. 6, pp. 841-846, 2005.

[11] T. Chen, W. Wu, and W. Zhou, "Global $\mu$-synchronization of linearly coupled unbounded time-varying delayed neural networks with unbounded delayed coupling," IEEE Transactions on Neural Networks, vol. 19, no. 10, pp. 1809-1816, 2008.

[12] Y. Wang, Z. Wang, and J. Liang, "Global synchronization for delayed complex networks with randomly occurring nonlinearities and multiple stochastic disturbances," Journal of Physics A, vol. 42, no. 13, Article ID 135101, 2009.

[13] K. Wang, X. Fu, and K. Li, "Cluster synchronization in community networks with nonidentical nodes," Chaos. An Interdisciplinary Journal of Nonlinear Science, vol. 19, no. 2, Article ID 023106, p. 10, 2009.

[14] J. Wang, J. Feng, C. Xu, and Y. Zhao, "Cluster synchronization of nonlinearly-coupled complex networks with nonidentical nodes and asymmetrical coupling matrix," Nonlinear Dynamics, vol. 67, pp. 1635-1646, 2012.

[15] V. Afraimovich, A. Cordonet, and N. F. Rulkov, "Generalized synchronization of chaos in noninvertible maps," Physical Review E, vol. 66, no. 1, Article ID 016208, 6 pages, 2002.

[16] S. Taherion and Y. C. Lai, "Observability of lag synchronization of coupled chaotic oscillators," Physical Review E, vol. 59, no. 6, pp. R6247-R6250, 1999.

[17] Q. Zhang and J. Zhao, "Projective and lag synchronization between general complex networks via impulsive control," Nonlinear Dynamics, vol. 67, pp. 2519-2525, 2011.

[18] M. G. Rosenblum, A. S. Pikovsky, and J. Kurths, "Phase synchronization of chaotic oscillators," Physical Review Letters, vol. 76, no. 11, pp. 1804-1807, 1996.

[19] R. Brown and L. Kocarev, "A unifying definition of synchronization for dynamical systems," Chaos, vol. 10, no. 2, pp. 344-349, 2000.

[20] D. Miller, K. Kowalski, and A. Lozowski, "Synchronization and anti-synchronization of Chua's oscillators via a piecewise linearcoupling circuit," Chaos, vol. 5, pp. 458-462, 1999.

[21] I. Wedekind and U. Parlitz, "Experimental observation of synchronization and anti-synchronization of chaotic lowfrequency-fluctuations in external cavity semiconductor lasers," International Journal of Bifurcation and Chaos in Applied Sciences and Engineering, vol. 11, no. 4, pp. 1141-1147, 2001. 
[22] J. Hu, S. Chen, and L. Chen, "Adaptive control for antisynchronization of Chua's chaotic system," Physics Letters A, vol. 339, no. 6, pp. 455-460, 2005.

[23] L. Hui, "Synchronization and anti-synchronization of Colpitts oscillators using active control," Chaos, Solitons and Fractals, vol. 26, no. 1, pp. 87-93, 2005.

[24] W. L. Li, X. Q. Chen, and Z. P. Shen, "Anti-synchronization of two different chaotic systems," Physica A, vol. 387, no. 14, pp. 3747-3750, 2008.

[25] C. Li and X. Liao, "Anti-synchronization of a class of coupled chaotic systems via linear feedback control," International Journal of Bifurcation and Chaos in Applied Sciences and Engineering, vol. 16, no. 4, pp. 1041-1047, 2006.

[26] J. Meng and X. Wang, "Robust anti-synchronization of a class of delayed chaotic neural networks," Chaos. An Interdisciplinary Journal of Nonlinear Science, vol. 17, no. 2, Article ID 023113, p. 6, 2007.

[27] H. Zhu and B. T. Cui, "The antisynchronization of a class of chaotic delayed neural networks," Chaos, vol. 17, no. 4, Article ID 043122, p. 7, 2007.

[28] H. Rui and A. Li, "A special full-state hybrid projective synchronization in symmetrical chaotic systems," Applied Mathematics and Computation, vol. 200, no. 1, pp. 321-329, 2008.

[29] H.-T. Yau, "Synchronization and anti-synchronization coexist in two-degree-of-freedom dissipative gyroscope with nonlinear inputs," Nonlinear Analysis. Real World Applications, vol. 9, no. 5, pp. 2253-2261, 2008.

[30] J. H. Chen, H. K. Chen, and Y. K. Lin, "Synchronization and anti-synchronization coexist in Chen-Lee chaotic systems," Chaos, Solitons and Fractals, vol. 39, no. 2, pp. 707-716, 2009.

[31] C. Grabow, S. Grosskinsky, and M. Timme, "Small-world network spectra in mean-field theory," Physical Review Letters, vol. 108, Article ID 218701, 2012.

[32] W. Sun, Z. Chen, Y. Lü, and S. Chen, "An intriguing hybrid synchronization phenomenon of two coupled complex networks," Applied Mathematics and Computation, vol. 216, no. 8, pp. 23012309, 2010.

[33] J. Lu and D. W. C. Ho, "Stabilization of complex dynamical networks with noise disturbance under performance constraint," Nonlinear Analysis. Real World Applications, vol. 12, no. 4, pp. 1974-1984, 2011.

[34] J. Feng, J. Wang, C. Xu, and F. Austin, "Cluster synchronization of nonlinearly coupled complex networks via pinning control," Discrete Dynamics in Nature and Society, Article ID 262349, 23 pages, 2011.

[35] J. Lu, J. Kurths, J. Cao, N. Mahdavi, and C. Huang, "Synchronization control for nonlinear stochastic dynamical networks: pinning impulsive strategy," IEEE Transactions on Neural Networks and Learning Systems, vol. 23, pp. 285-291, 2012.

[36] X. Liu and T. Chen, "Synchronization analysis for nonlinearlycoupled complex networks with an asymmetrical coupling matrix," Physica A, vol. 387, no. 16-17, pp. 4429-4439, 2008.

[37] X. Liu and T. Chen, "Synchronization of identical neural networks and other systems with an adaptive coupling strength," International Journal of Circuit Theory and Applications, vol. 38, no. 6, pp. 631-648, 2010.

[38] W. Wu and T. Chen, "Global synchronization criteria of linearly coupled neural network systems with time-varying coupling," IEEE Transactions on Neural Networks, vol. 19, no. 2, pp. 319332, 2008. 


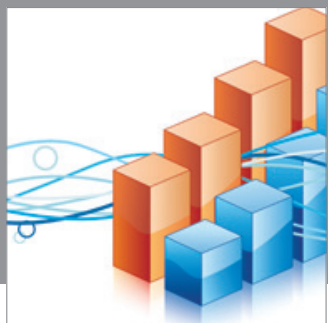

Advances in

Operations Research

mansans

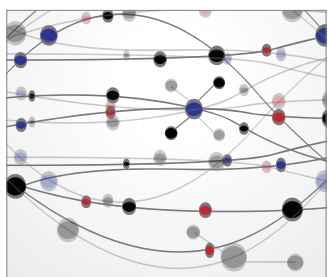

The Scientific World Journal
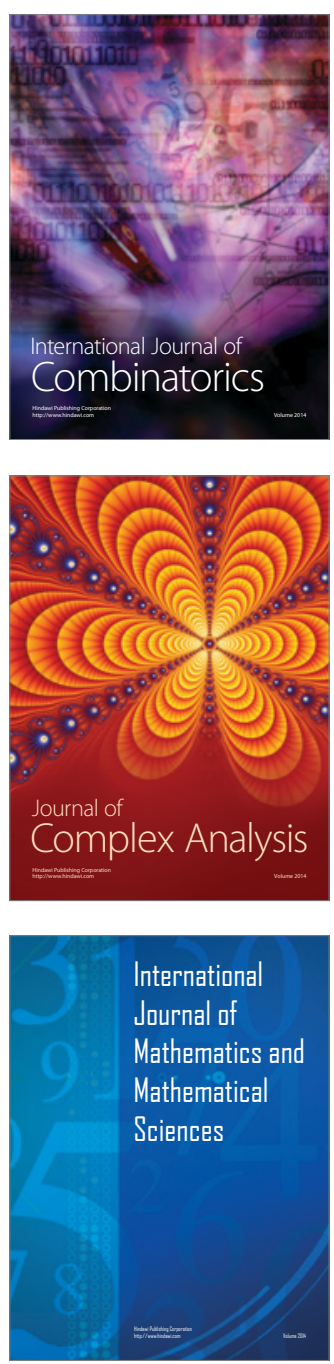
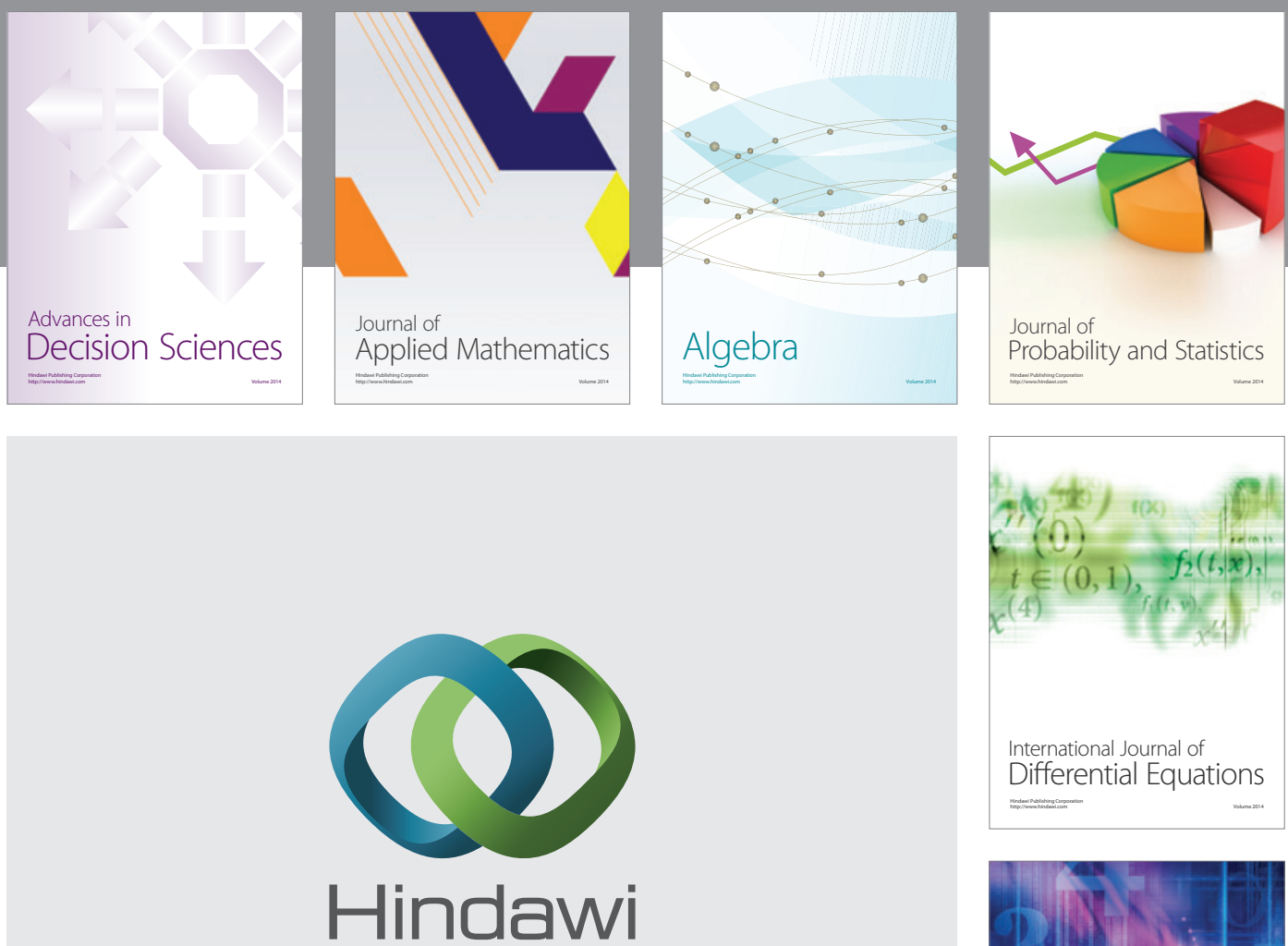

Submit your manuscripts at http://www.hindawi.com
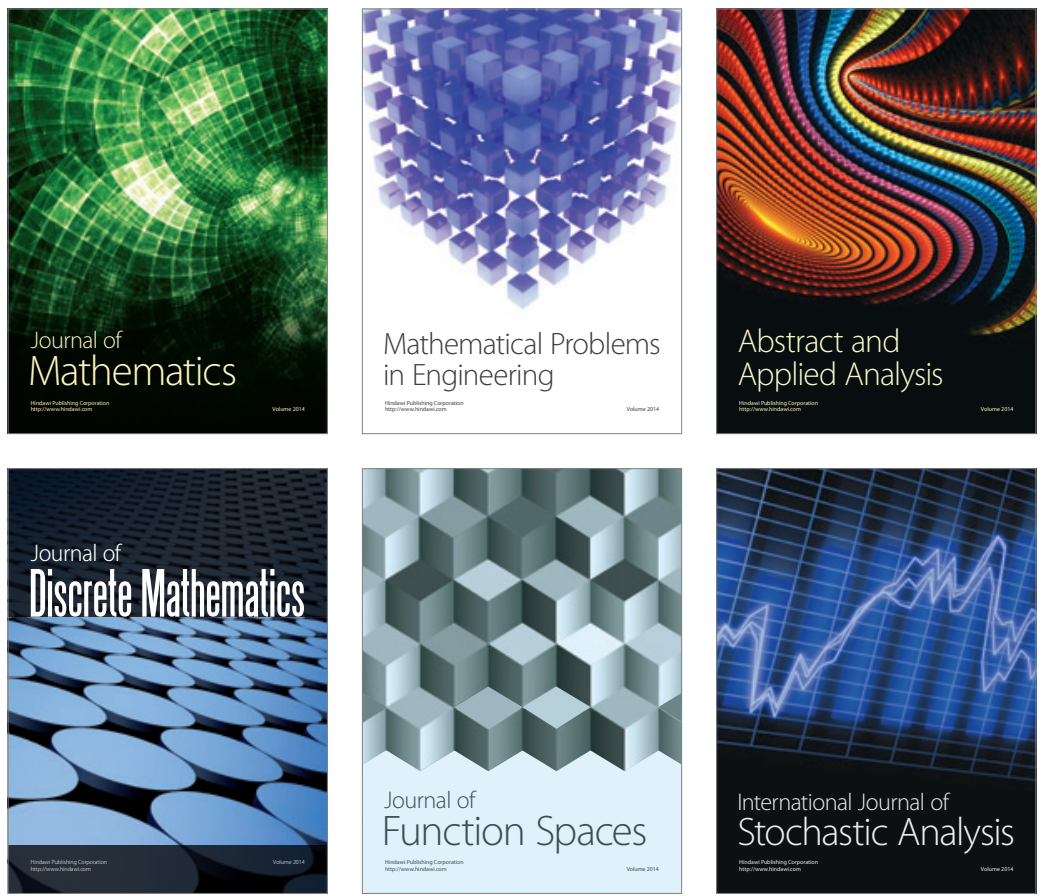

Journal of

Function Spaces

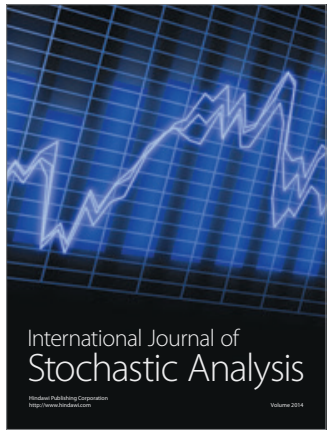

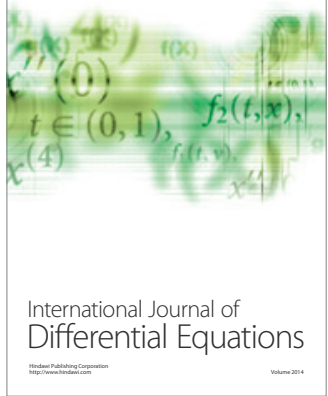
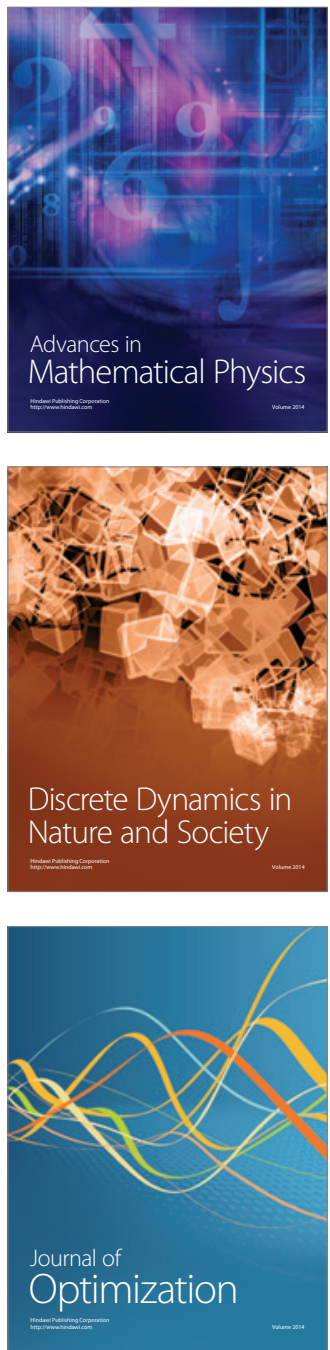